\title{
A production scenario of the galactic strangelets and an estimation of their possible flux in the solar neighborhood
}

\author{
Sayan Biswas ${ }^{a, b, 1 *}$, Arunava Bhadra ${ }^{c, 2}$, J.N. De ${ }^{d, 3}$, Partha S. Joarder ${ }^{a, b, 4}$, Sibaji \\ Raha $^{a, b, 5}$ and Debapriyo Syam ${ }^{b, 6}$ \\ a Department of Physics, Bose Institute \\ 93/1 A.P.C. Road, Kolkata 700009, India \\ ${ }^{b}$ Centre for Astroparticle Physics and Space Science, Bose Institute \\ Block EN, Sector V, Salt Lake, Kolkata 700091, India \\ ${ }^{c}$ High Energy \& Cosmic Ray Research Centre, University of North Bengal \\ Siliguri 734013, West Bengal, India \\ ${ }^{d}$ Saha Institute of Nuclear Physics \\ 1/AF, Bidhannagar, Kolkata 700064, India \\ E-mail: ${ }^{1}$ sayan@jcbose.ac.in, ${ }^{2}$ aru_bhadra@yahoo.com, ${ }^{3} j$ n.de@saha.ac.in, \\ ${ }^{4}$ parthaljcbose.ac.in, ${ }^{5}$ sibaji.raha@jcbose.ac.in, \\ ${ }^{6}$ syam. debapriyo@gmail.com
}

Finite lumps of strange quark matter in the form of strangelets, the theorized absolute ground state of QCD, are supposed to be more stable than ${ }^{56} \mathrm{Fe}$ nuclei. Recent simulation studies suggest that a major source of strangelets in the Galaxy may be the fragmentation of the bulk strange matter which is tidally released in the merger of strange stars in compact binary stellar systems. Here, we determine a plausible baryon number (or mass) distribution of such strangelets by invoking a statistical disassembly model often used in nuclear systems. The produced strangelets are likely to be accelerated by the shock front generated in the stellar merger itself thereby gaining a power law energy spectrum with spectral index close to -2 . We estimate the fluxes of such accelerated strangelets of different masses in the neighborhood of the Sun considering diffusive propagation of strangelets from the sources in the stochastic magnetic field of the interstellar medium. The determined fluxes are found to be consistent with the null results of the PAMELA experiment. The reported limit of sensitivity of the AMS-02 experiment suggests that the experiment should be able to detect strangelet events, as per the prediction of the proposed model, thereby (possibly) vindicating the strange matter hypothesis.

The 34th International Cosmic Ray Conference, 30 July- 6 August, 2015

The Hague, The Netherlands

* Speaker. 


\section{Introduction}

According to the strange matter hypothesis (SMH) [1], strange quark matter (SQM), containing almost equal numbers of up (u-), down (d-) and strange (s-) quarks enclosed in a MIT bag [2] simulating the spatial and color confinement of the quarks, may be the true ground state of hadronic matter. Despite finite size effects, small lumps of SQM (or strangelets), with suitable bag parameters, can also be more stable than ${ }^{56} \mathrm{Fe}$ nuclei [3]. Recent theoretical and simulation studies suggest that the fragmentation of the globally charge-neutral (due to the presence of electrons inside) bulk SQM ejected in the merger of strange stars (SSs) in the binary stellar systems in the galaxy and the eventual establishment of the thermodynamic and chemical equilibrium in that fragmenting system would be a possible scenario for the formation of the galactic strangelets $[4,5,6,7,8]$ that contribute to the primary cosmic rays (PCR). The aim of this work is to derive a plausible mass spectrum of strangelets at the site of SS merger by invoking a statistical multifragmentation model (SMM) [9]. In this paper, we further predict a strangelet flux in the solar neighborhood by considering the diffusive propagation of strangelets, originating at the site of SS merger, in the stochastic magnetic field of the interstellar medium. Such estimation of the strangelet flux may be important in view of the ongoing efforts to detect strangelets in PCR by the PAMELA [10] and the AMS-02 [11] experiments.

\section{Statistical multifragmentation model}

We assume that the fragmenting SQM (hereafter referred to as the strangelet-complex) eventually occupies a freeze-out volume in thermodynamic and chemical equilibrium at a constant temperature $(T)$. The strong interactions between the strangelet-fragments within the complex are assumed to cease to exist at such freeze-out. The strangelet-complex is assumed to be a bulk matter with a large but finite volume; finite size effects of its surface and curvature are ignored. Global charge neutrality of the strangelet-complex (in equilibrium) requires that each of the positively charged strangelet-fragments in that complex is surrounded by a charge-neutralizing electron cloud whose volume is much larger than the volume of the fragment. The condition of equilibrium of the strangelet-complex necessitates that the electron density $\left(n_{e}\right)$ and the electron chemical potential $\left(\mu_{e}=\left(3 \pi^{2} n_{e}\right)^{1 / 3}\right)$ are approximately constant in that complex. Here, we assume $\mu_{e} \approx \frac{m_{s}^{2}}{4 \mu_{q}}[12,13,8]$ (which is in fact the expression appropriate for a globally charge-neutral bulk SQM at zero-pressure and zero-temperature with $\mu_{q}^{2} \gg m_{s}^{2}$ ) for the sake of simplicity; $m_{s}$ being the current-mass of the $s$ - quarks and $\mu_{q}$ being the constant quark number chemical potential of the strangelet-complex, with a constant baryon number $A_{b}$, in thermodynamic equilibrium at freezeout. Chemical equilibrium in the strangelet-complex, established by the weak interaction processes $\mu_{f}=\mu_{q}-q_{f} \mu_{e},\left(f=u, d, s ; q_{f}\right.$ being the charge of the corresponding quark-flavor in the unit of the magnitude of the electronic charge $e$ ) further demands that the chemical potentials ' $\mu_{f}$ 's of the individual quark-flavors are constants throughout the volume of that strangelet-complex at freezeout [12].

The multiplicity $\omega^{i}$ of the strangelets of species ' $i$ ', that is characterized by its baryon number $A^{i}$, is written as $[6,7,8,9]$ 


$$
\omega^{i}=\frac{\mathscr{V}}{\left(\mathscr{L}^{i}\right)^{3}} e^{\left(\mu^{i}-F^{i}\right) / T} .
$$

Here, $\mathscr{V}$ is the available volume, ie. the freeze-out volume minus the volume of the produced fragments. In Eq. (2.1), $\mu^{i}=\sum_{(f, e)} \mu_{(f, e)} N_{(f, e)}^{i}$ is the chemical potential of the $i^{\text {th }}$ species with $N_{(f, e)}^{i}$ $=\left(-\frac{\partial \Omega_{(f, e)}^{i}}{\partial \mu_{(f, e)}}\right)_{V^{i}, T}$ being either the number of quarks of the $f^{\text {th }}$ flavor $(f=u, d, s)$ or the number of electrons in a fragment of the $i^{\text {th }}$ species of volume $\mathrm{V}^{i}$; their thermodynamic potentials are $\Omega_{(f, e)}^{i}$. The thermal de-Broglie wavelength of a strangelet of the $i^{\text {th }}$ species is defined as $\mathscr{L}^{i}=h / \sqrt{2 \pi m^{i} T}$ where $m^{i}$ is the mass of the strangelet $[4,8]$. Here, $F^{i}\left(=\Omega^{i}+\mu^{i}+E_{\mathrm{C}}^{i}\right)$ stands for the Helmholtz free energy of the $i^{\text {th }}$ species while $\Omega^{i}$ is its thermodynamic potential and $E_{\mathrm{C}}^{i}$ is its Coulomb energy. $F^{i}$ may be rewritten as $F^{i}=\Omega_{\text {tot }}^{i}+\mu^{i}$, where, $\Omega_{\mathrm{tot}}^{i}=\Omega^{i}+E_{\mathrm{C}}^{i}$. Thus, Eq. (2.1) can be reframed as

$$
\omega^{i}=\frac{\mathscr{V}}{\left(\mathscr{L}^{i}\right)^{3}} e^{-\Omega_{\mathrm{tot}}^{i} / T} .
$$

We will use Eq. (2.2) to determine the multiplicities of various fragments in the strangelet complex. Throughout this work, we use natural units such that $\hbar=c=k_{\mathrm{B}}=1$ and $\alpha=\frac{1}{137}$, where $k_{\mathrm{B}}$ is the Boltzmann constant and $\alpha$ is the fine structure constant.

\section{Thermodynamical properties of strangelet}

The radius of an assumed spherical strangelet of the $i^{\text {th }}$ species is $R^{i}=r_{\mathrm{o}}^{i}\left(A^{i}\right)^{1 / 3}, r_{\mathrm{o}}^{i}$ being its radius parameter. Volume, surface and curvature of a strangelet are denoted as $\mathrm{V}^{i}=\frac{4}{3} \pi\left(R^{i}\right)^{3}$, $\mathrm{S}^{i}=4 \pi\left(R^{i}\right)^{2}$ and $C^{i}=8 \pi R^{i}$, respectively. Thermodynamic potential of the $i^{\text {th }}$ species is written as $\Omega^{i}=\Omega_{\mathrm{V}}^{\mathrm{o}} \mathrm{V}^{i}+\Omega_{\mathrm{S}}^{\mathrm{o}} \mathrm{S}^{i}+\Omega_{\mathrm{C}}^{\mathrm{o}} \mathrm{C}^{i}+B \mathrm{~V}^{i}$.

Here, $\Omega_{V}^{o}, \Omega_{\mathrm{S}}^{\mathrm{o}}$ and $\Omega_{\mathrm{C}}^{\mathrm{o}}$ are the thermodynamic potential densities associated with the volume, surface and curvature of the strangelets. The expressions for these quantities are [8]:

$$
\begin{array}{r}
\Omega_{\mathrm{V}}^{\mathrm{o}}=-\frac{37}{90} \pi^{2} T^{4}-\left(\frac{\mu_{u}^{2}+\mu_{d}^{2}}{2}\right) T^{2}-\left(\frac{\mu_{u}^{4}+\mu_{d}^{4}}{4 \pi^{2}}\right)-\frac{\mu_{s}^{4}}{4 \pi^{2}}\left[\left(1-\frac{5}{2} \lambda_{s}^{2}\right) \sqrt{1-\lambda_{s}^{2}}+\frac{3}{2} \lambda_{s}^{4} \ln \left(\frac{1+\sqrt{1-\lambda_{s}^{2}}}{\lambda_{s}}\right)\right. \\
\left.+2 \pi^{2}\left(\frac{T}{\mu_{s}}\right)^{2} \sqrt{1-\lambda_{s}^{2}}+\frac{7 \pi^{4}}{15}\left(\frac{T}{\mu_{s}}\right)^{4} \frac{\left(1-\frac{3}{2} \lambda_{s}^{2}\right)}{\left(1-\lambda_{s}^{2}\right)^{3 / 2}}\right]-\frac{\mu_{e}^{4}}{12 \pi^{2}},
\end{array}
$$

$$
\begin{aligned}
\Omega_{\mathrm{S}}^{\mathrm{o}}=\frac{3}{4 \pi} \mu_{s}^{3}\left[\frac{\left(1-\lambda_{s}^{2}\right)}{6}-\frac{\lambda_{s}^{2}}{3}\left(1-\lambda_{s}\right)-\right. & \frac{1}{3 \pi}\left\{\tan ^{-1}\left(\frac{\sqrt{1-\lambda_{s}^{2}}}{\lambda_{s}}\right)+\lambda_{s}^{3} \ln \left(\frac{1+\sqrt{1-\lambda_{s}^{2}}}{\lambda_{s}}\right)-2 \lambda_{s} \sqrt{1-\lambda_{s}^{2}}\right\} \\
& \left.+\frac{\pi}{3}\left(\frac{T}{\mu_{s}}\right)^{2}\left\{\frac{\pi}{2}-\tan ^{-1}\left(\frac{\sqrt{1-\lambda_{s}^{2}}}{\lambda_{s}}\right)\right\}+\frac{7 \pi^{3}}{180}\left(\frac{T}{\mu_{s}}\right)^{4} \frac{\lambda_{s}^{3}}{\left(1-\lambda_{s}^{2}\right)^{3 / 2}}\right]
\end{aligned}
$$

and 


$$
\begin{aligned}
\Omega_{\mathrm{C}}^{\mathrm{o}}=\frac{19}{36} T^{2} & +\left(\frac{\mu_{u}^{2}+\mu_{d}^{2}}{8 \pi^{2}}\right)+\frac{\mu_{s}^{2}}{8 \pi^{2}}\left[\frac{1}{\lambda_{s}}\left\{\frac{\pi}{2}-\tan ^{-1}\left(\frac{\sqrt{1-\lambda_{s}^{2}}}{\lambda_{s}}\right)\right\}+\left(\frac{\pi^{2}}{\lambda_{s}}\right)\left(\frac{T}{\mu_{s}}\right)^{2}\left\{\frac{\pi}{2}-\tan ^{-1}\left(\frac{\sqrt{1-\lambda_{s}^{2}}}{\lambda_{s}}\right)\right\}\right. \\
& \left.+\lambda_{s}^{2}\left\{\pi+\ln \left(\frac{1+\sqrt{1-\lambda_{s}^{2}}}{\lambda_{s}}\right)\right\}-\frac{3 \pi}{2} \lambda_{s}-\left(\frac{2 \pi^{2}}{3}\right)\left(\frac{T}{\mu_{s}}\right)^{2} \frac{1}{\sqrt{1-\lambda_{s}^{2}}}-\frac{7 \pi^{4}}{60}\left(\frac{T}{\mu_{s}}\right)^{4} \frac{\lambda_{s}^{2}\left(1+\lambda_{s}^{2}\right)}{\left(1-\lambda_{s}^{2}\right)^{5 / 2}}\right] .
\end{aligned}
$$

In the above, $\lambda_{s}=\frac{m_{s}}{\mu_{s}}$. The net positive charge density inside a strangelet is confined within a layer of thickness $\sim \lambda_{D}\left(\approx \frac{7.33}{\mu_{q}} ; \lambda_{D}\right.$ is the Debye screening length) from its surface. In this situation, the total charge $\left(Z^{i}\right)$ and the Coulomb energy of the strangelet is obtained by integrating over the radial coordinate $(r)$ measured from its centre. The expressions of these integrated quantities are obtained from Refs. [13, 14]. The total energy of a strangelet can be written as $E^{i}=T \mathscr{S}^{i}+\mu^{i}+\Omega_{\mathrm{tot}}^{i}=$ $T \mathscr{S}^{i}+\mu^{i}+\Omega^{i}+E_{\mathrm{C}}^{i}$, where, $\mathscr{S}^{i}=-\left(\frac{\partial \Omega^{i}}{\partial T}\right)_{\mathrm{V}^{i}, \mu^{i}}$ is the entropy of $i^{\text {th }}$ species. The strangelet fragments are also in mechanical equilibrium, ie. $P_{\mathrm{ext}}^{i}=-\left(\frac{\partial \Omega_{\mathrm{tot}}^{i}}{\partial \mathrm{V}^{i}}\right)_{T, \mu^{i}} ; P_{\mathrm{ext}}^{i}$ being the external pressure on a strangelet of the $i^{\text {th }}$ species. This pressure is assumed here to be exerted by $Z^{i}$ charge-neutralizing relativistic electrons residing outside the $i^{\text {th }}$ fragment but within the WignerSeitz (WS) cell surrounding that particular fragment. Here, the strangelet fragment of the $i^{\text {th }}$ species is approximated to be a pointlike positive charge $\left(Z^{i}\right)$ surrounded by a spherical WS cell containing electrons of uniform number density [15] $\frac{Z^{i}}{V_{\text {cell }}^{i}}=\frac{N_{e}^{\text {total }}}{\mathscr{V}}=n_{e}=\frac{m_{s}^{6}}{192 \pi^{2} \mu_{q}^{3}} ; V_{\text {cell }}^{i}$ is the volume of the WS cell, $N_{e}^{\text {total }}$ is the total number of electrons in the strangelet complex. We choose $V_{\text {cell }}^{i}=\frac{Z^{i}}{\sum_{i} Z^{i} \omega^{i}} \mathscr{V}$ so that it satisfies the constraint $\sum_{i} \omega^{i} V_{\text {cell }}^{i}=\mathscr{V}$. The expression for the pressure of the relativistic electrons on the strangelet may then be written as [15]

$$
\begin{aligned}
& P_{\mathrm{ext}}^{i}=\left(3 \pi^{2}\right)^{1 / 3}\left[\frac{\left(n_{\mathrm{e}}\right)^{4 / 3}}{4}\right]+\left(\frac{\pi^{2}}{2}\right)\left[\frac{T^{2}}{\left(3 \pi^{2}\right)^{1 / 3}}\right]\left(n_{\mathrm{e}}\right)^{2 / 3} \\
& -\left(\frac{3}{10}\right)\left(\frac{4 \pi}{3}\right)^{1 / 3} \alpha\left(Z^{i}\right)^{2 / 3}\left(n_{e}\right)^{4 / 3} \\
& -\left(\frac{1}{6}\right)\left(\frac{324}{175}\right)\left(\frac{4}{9 \pi}\right)^{2 / 3}\left(3 \pi^{2}\right)^{1 / 3}\left(Z^{i}\right)^{4 / 3} \alpha^{2}\left(n_{e}\right)^{4 / 3} \\
& +\left(\frac{1}{8 \pi}\right) \alpha\left(3 \pi^{2}\right)^{1 / 3}\left(n_{e}\right)^{4 / 3}-\frac{0.062}{6} \alpha^{2} m_{e} n_{e} .
\end{aligned}
$$

Here, $m_{e}$ is the mass of the electron. We further require an additional relation $N_{u}^{i}=A^{i}+Z^{i}$, that is obtained from the definitions of the baryon number and the charge of a strangelet [8].

\section{Mass spectra of strangelets}

In this section, our aim is to numerically determine the baryon number (or size) distribution of various species of strangelets in the fragmenting complex in thermodynamic equilibrium at freezeout. For that purpose we need to first examine the condition of global charge-neutrality in the strangelet complex. This condition is written as 


$$
\sum_{i} \frac{\omega^{i}}{\mathscr{V}} Z^{i}=\sum_{i} n^{i} Z^{i}=n_{e}=\frac{m_{s}^{6}}{192 \pi^{2} \mu_{q}^{3}}
$$

with $n^{i}=\frac{\omega^{i}}{\mathscr{V}}$ being defined as the multiplicity density of strangelet-fragments of the $i^{\text {th }}$ species. In the next step, we are required to determine the value of the available volume $\mathscr{V}$ of the strangelet complex in equilibrium at a certain temperature at freeze-out. This is done by using the condition for conservation of the initial baryon number $A_{b}[6,7]$ of the strange matter released in an SS merger event. This condition may be written in a form

$$
\mathscr{V}=\frac{A_{b}}{\sum_{i} A^{i} n^{i}}
$$

The value of the multiplicity $\omega^{i}$ of strangelets of the $i^{\text {th }}$ species may now be easily determined from the known values of $n^{i}$ and $\mathscr{V}$. For the baryon number of the initial bulk matter, we choose $A_{b}=$ $1 \times 10^{53}$; this corresponds to a population averaged tidally released mass $M_{\text {ejected }} \approx 10^{-4} M_{\odot}$ [5] per binary SS merger obtained in the simulations with a bag value that corresponds to $B^{1 / 4} \approx 145 \mathrm{MeV}$.
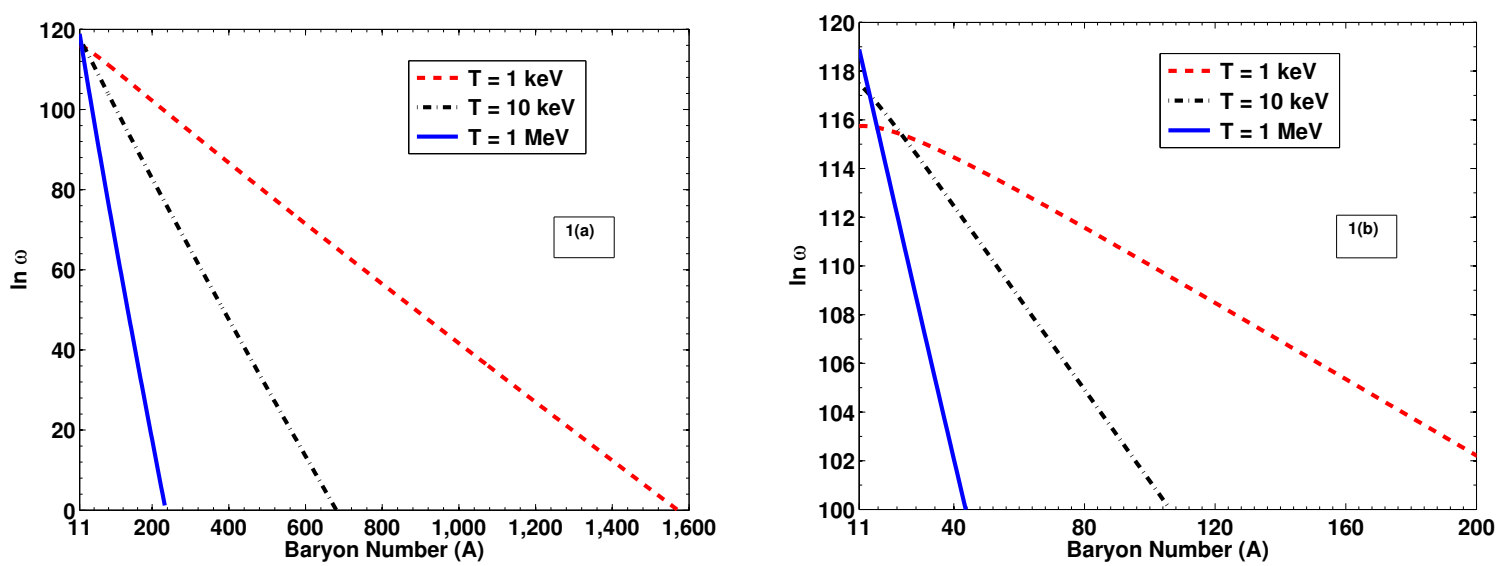

Figure 1: (color online) $\ln \omega$ vs. $A$ for the strangelet fragments with $B^{1 / 4}=145 \mathrm{MeV}$ and $m_{s}=95 \mathrm{MeV}$ [16] at three different temperatures at freeze-out. Variations are displayed for (a) the full range of possible baryon numbers and for (b) a limited range of baryon numbers of the fragments. Available volume is determined to be $\mathscr{V} \approx 4 \times 10^{50} \mathrm{MeV}^{-3}, A_{\mathrm{b}}=1 \times 10^{53}$.

Figs. 1(a,b) display the size distributions of strangelet fragments for a fixed bag value $\left(B^{1 / 4}=\right.$ $145 \mathrm{MeV}$ ) at three different temperatures, namely $T=1 \mathrm{keV}, T=10 \mathrm{keV}$ and $T=1 \mathrm{MeV}$, respectively. The variation of size distribution with changing temperature is in qualitative agreement with the one obtained in Ref. [6] in the case of massless quarks. Suppression of heavier fragments and enhanced production of lighter fragments with increasing temperature are noted for $m_{s}=0[6]$ and also for $m_{s} \neq 0[7,8]$. The results are also in consonance with those obtained in nuclear multifragmentation [9, 17]. Recent results in Ref. [18] show opposite pattern for color flavor locked (CFL) strangelets. It is however interesting to note that fragmentation pattern as shown in our present work is similar to our previous investigation for CFL case (for $m_{s}=0$ ) [19]. 


\section{An estimation of flux of strangelets}

We here, shall estimate the flux of the strangelets, originated from binary strange star mergers, at the top of the atmosphere. Being charged particles, the produced strangelets will undergo Fermi acceleration by interacting with the shocks produced either in strange star collisions and/or when the ejected mass slows down by colliding with the surrounding medium. The generated shocks are likely to be relativistic as indicated by the gamma ray bursts observations which are believed to occur in mergers of two super dense stellar objects. Typically $10 \%$ of the total shock energy is used for particle acceleration [20]. The resultant energy spectrum of all strangelets (sum of strangelets of different baryon numbers, similar to all particle cosmic rays) will be a power law $d N / d \mathscr{E}=\mathscr{N}_{o} \mathscr{E}^{-\alpha}$, where $\mathscr{N}_{o}$ is the normalization constant, $\mathscr{E}$ is the kinetic energy and $\alpha$ is the spectral index which is taken here as 2.2 [4].

The age of our galaxy (Milky Way) may be considered to be much larger than the time scale for strangelet acceleration, energy loss, spallation and escape from the Galaxy and due to this, it is reasonable to assume that the astrophysical strangelets obey a steady state distribution [4]. Our Galaxy can be roughly described as a cylindrical disc having thickness $300 \mathrm{pc}$ and radius $20 \mathrm{kpc}$. Since the width of our Galaxy is much smaller than its radius $\left(R_{d}\right)$ we may consider the disc as infinitely thin in the $\mathrm{z}$ direction i.e. the sources are all located in a plane with $\mathrm{z}=0$. Due to lack of observational/experimental data it is difficult to describe the source distribution of binary strange stars. For simplicity, we consider that such sources are uniformly distributed over the galactic disc. If the shock energy is $\sim 10^{49} \operatorname{ergs}$ [21] the differential flux for $i^{\text {th }}$ species (with $\frac{E^{i}}{A^{i}}<930 \mathrm{MeV}$ ) is

$$
\phi^{i}\left(\mathscr{R}^{i}\right)=4.6 \times 10^{6} \frac{\omega^{i}}{\sum_{i} \varepsilon_{\min }^{i} \omega^{i}}\left(\beta^{i}\right)^{2}\left(Z^{i}\right)^{\frac{4}{3}}\left(\eta^{i}\right)^{-2.53} \mathrm{~m}^{-2} \mathrm{sr}^{-1} \mathrm{yr}^{-1} \mathrm{GV}^{-1},
$$

where, $\varepsilon_{\text {min }}^{i}=\frac{1}{2} m_{0}^{i} \beta_{\text {min }}^{2}, m_{0}^{i}$ being the rest mass energy $\left(m_{0}^{i}=E^{i}\right)$ of $i^{t h}$ species and $\beta_{\text {min }}=0.15$ [21]. Binary SS merger rate is taken to be $10^{-5} \mathrm{yr}^{-1}$ [4]. Rigidity for a particular species is $\mathscr{R}^{i}=\frac{m_{0}^{i}}{Z^{i}} \frac{\beta^{i}}{\sqrt{1-\left(\beta^{i}\right)^{2}}}$ and $\eta^{i}=\left(\frac{\mathscr{R}^{i} Z^{i}}{\beta^{i}}-m_{0}^{i}\right)$. For a particular species, $\mathscr{R}^{i}$ is varied and $\beta^{i}$ is calculated depending on $\mathscr{R}^{i}$ and $Z^{i}$. We used the energy dependent diffusion coefficient, $D^{i}=1.4 \times$ $10^{28}\left(\frac{\mathscr{E}^{i}}{1 \mathrm{GV} Z^{i}}\right)^{1 / 3} \mathrm{~cm}^{2} / \mathrm{s}[22], \mathscr{E}^{i}$ being the kinetic energy of $i^{t h}$ species and $\mathscr{E}^{i}=\sqrt{\left(\mathscr{R}^{i}\right)^{2}\left(Z^{i}\right)^{2}+\left(m_{0}^{i}\right)^{2}}-$ $m_{0}^{i}$. Though Eq. (5.1) is calculated as the flux at the centre of the disc due to all sources in the galactic plane but due to diffusion over a large period of time a homogeneous distribution of strangelets is expected so that it has equal flux at each point in the disc plane. The differential flux obtained by our approach is compared in figs. 2(a,b) with the upper limit of flux as obtained by PAMELA [10] and the best fitted lines are shown. The theoretical differential flux for $A^{i}=11, Z^{i}=1$ and $A^{i}=30, Z^{i}=2$ are found well below the upper limits of flux obtained by PAMELA. We have also checked the differential fluxes for the same $A^{i}, Z^{i}$ sets at two extreme temperatures ( $\mathrm{T}=1 \mathrm{MeV}$ and $\mathrm{T}=1 \mathrm{keV}$ ) and those are also below the upper limits. Hence, our results justify the null results as obtained by PAMELA experiment [10]. The present model can be tested experimentally if future experiments can improve the flux limit by an order or two, particularly at lower rigidity end. In the flux calculation solar modulation is not incorporated. That can be done by the prescription suggested in the Ref. [23] which will effectively lower the flux at low rigidity end. Further considering the fact that the PAMELA observations were mostly conducted in minimum solar activity time cycle, the Eq. (5.1) gives a reasonable approximation of the flux even at lower rigidity end. 

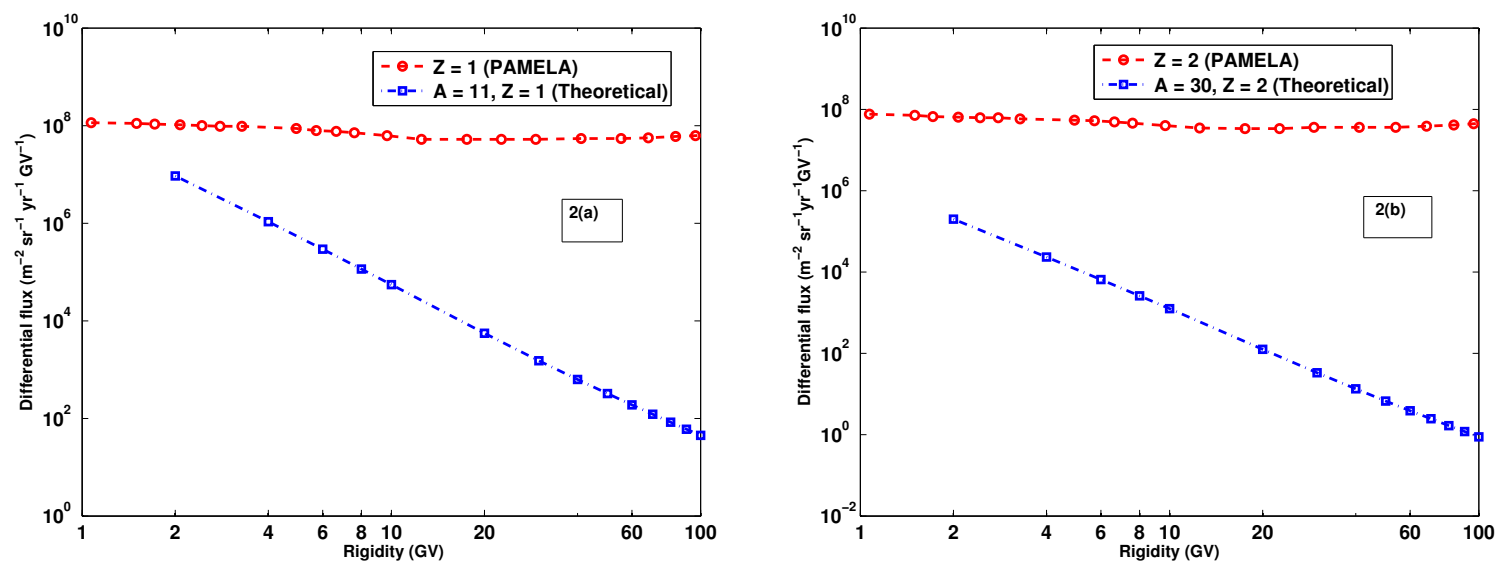

Figure 2: (color online) Differential flux vs. rigidity for the strangelet fragments with $B^{1 / 4}=145 \mathrm{MeV}$ and $m_{s}=95 \mathrm{MeV}$ [16] at $\mathrm{T}=10 \mathrm{keV}$ at freeze-out. Comparisons with PAMELA results are displayed for (a) $A=11, Z=1$ and for (b) $A=30, Z=2$. Available volume is determined to be $\mathscr{V} \approx 4 \times 10^{50} \mathrm{MeV}^{-3}$, $A_{\mathrm{b}}=1 \times 10^{53}$ and $\beta^{i}=\frac{v^{i}}{c}, v^{i}$ is the velocity of $i^{\text {th }}$ species.

\section{Conclusion}

In this work, we have tried to give a plausible production scenario of galactic strangelets. Possible strangelet mass spectrum is then obtained with the help of statistical multifragmentation model. Strangelets in that mass spectrum are then injected in shocks generated by binary SS mergers and gained a power law spectrum. The flux of strangelets at the solar neighborhood is estimated considering the diffusive shock acceleration of strangelets in strange stars merger environment. Our result is consistent with the null result as obtained by PAMELA experiment regarding strangelets flux. It is expected that present results will be useful for AMS-02 and other future experiments with more sensitive and sophisticated detectors those may detect strangelets and ultimately vindicate the SQM hypothesis.

\section{Acknowledgments}

JND acknowledges the support from the DST, Govt. of India. SR and PSJ thank the DST, Govt. of India for support under the IRHPA scheme.

\section{References}

[1] E. Witten, Cosmic separation of phases, Phys. Rev. D 30 (1984) 272.

[2] A. Chodos et al., New extended model of hadrons, Phys. Rev. D 9 (1974) 3471.

[3] E. Farhi and R.L. Jaffe, Strange matter, Phys. Rev. D 30 (1984) 2379.

[4] J. Madsen, Strangelet propagation and cosmic ray flux, Phys. Rev. D 71 (2005) 014026.

[5] A. Bauswein et al., Mass ejection by strange star mergers and observational implications, Phys. Rev. Lett. 103 (2009) 011101; A. Bauswein et al., Discriminating strange star mergers from neutron star mergers by gravitational wave measurements, Phys. Rev. D 81 (2010) 024012. 
[6] S. Biswas et al., Multifragmentation model for astrophysical strangelets, Phys. Lett. B715 (2012) 30.

[7] S. Biswas et al., Disassembly model for the production of astrophysical strangelets, Proc. Indian Natn. Sci. Acad. 81 (Issue 1) (2015) 277.

[8] S. Biswas et al., Multifragmentation model for the production of astrophysical strangelets, [arXiv:1409.8366v4 (2015)].

[9] J.P. Bondorf et al., Statistical multifragmentation of nuclei, Phys. Rep. 257 (1995) 133.

[10] M. Casolino et al. (PAMELA collaboration), New upper limit on strange quark matter flux with the PAMELA experiment, in Proc. 33rd International Cosmic Ray Conference, Rio de Janeiro, Brazil 2013, www.cbpf.br/ icrc2013/papers/icrc2013-1214.pdf.

[11] A. Kounine (AMS-02 Collaboration), Status of the AMS Experiment, in XVI International Symposium on Very High Energy Cosmic Ray Interactions (ISVHECRI 2010), Batavia, IL, USA. [arXiv:1009.5349v1].

[12] M. Alford and K. Rajagopal, Absence of two-flavor color-superconductivity in compact stars , JHEP 6 (2002) 31.

[13] M.G. Alford et al., Stability of strange star crusts and strangelets, Phys. Rev. D 73 (2006) 114016.

[14] H. Heiselberg, Screening in quark droplets, Phys. Rev. D 48 (1993) 1418.

[15] E.E. Salpeter, Energy and pressure of a zero-temperature plasma, ApJ 134 (1961) 669.

[16] J. Beringer et al. (Particle Data Group), Phys. Rev. D 86 (2012) 010001, http://pdg.lbl.gov/2013/tables/contents_tables.html; K.A. Olive et al. (Particle Data Group), Chin. Phys. C 38 (2014) 090001.

[17] D.H.E. Gross, Statistical decay of very hot nuclei-the production of large clusters, Rep. Prog. Phys. 53 (1990) 605; S. Pal et al., The effect of flow on nuclear multifragmentation in a quantum statistical model, Nucl. Phys. A608 (1996) 49.

[18] L. Paulucci and J.E. Horvath, Strange quark matter fragmentation in astrophysical events, Phys. Lett. B733 (2014) 164.

[19] S. Biswas et al., Quantum statistical multifragmentation model for the production of astrophysical strangelets, in Proc. 33rd International Cosmic Ray Conference, Rio de Janeiro, Brazil 2013, www.cbpf.br/ icrc2013/papers/icrc2013-0619.pdf.

[20] W.I. Axford et al., The acceleration of cosmic rays by shock waves, in Proc. 15th International Cosmic Ray Conference, Plovdiv, Bulgaria, 11 (1977) 132; E. A. Helder, et al., Cosmic-ray acceleration efficiency versus temperature equilibration: The case of SNR 0509-67.5, ApJ 719 (2010) L140, erratum ApJ 737 (2011) L46.

[21] K. Hotokezaka et al., The mass ejection from the merger of binary neutron stars, Phys. Rev. D 87 (2013) 024001.

[22] V.S. Berezinsky, Cosmic ray propagation in the galaxy, in R.J. Protheroe (Ed.), Proc. 21st International Cosmic Ray Conference, Adelaide, Australia, 11 (1990) 115.

[23] I. G. Usoskin et al., Heliospheric modulation of cosmic rays: Monthly reconstruction for 1951-2004, J. Geophys. Res. 110 (2005) A12108; A. Bhadra et al., Study of low energy hadronic interaction models based on BESS observed cosmic ray proton and antiproton spectra at medium high altitude, Phys. Rev. D. 79 (2009) 114027. 